\section{Comment on 'Postpartum contraception: a missed opportunity to prevent unintended pregnancy and short inter-pregnancy intervals'}

Heller et al. ${ }^{1}$ highlight the potential gap in providing contraception care postpartum. We also saw a missed opportunity to educate postnatal patients about contraception on our wards and to supply such care before discharge.

We work in a busy culturally and ethnically diverse area of outer London, with 5300 deliveries annually. We carried out a short face-to-face survey of 50 women who had delivered during the previous week. Twenty-two of the women did not have English as their first language. Their median age was 30 (range 17-43) years, with 36 having a higher education qualification. Average parity following the index pregnancy was two, with 32 women having delivered vaginally and 16 having had caesarean section and two an instrumental delivery.

We asked what method of contraception the women had used previously. Eighteen had not used any. Nine had used barrier methods and 15 either the progestogen-only pill or the combined pill or patch. Only eight (16\%) women had used long-acting reversible contraception (LARC), well below the country average of $37 \% .^{2}$

During the index pregnancy, only six women had discussed future contraception options with a member of staff, though 39 said that they were not considering a further pregnancy in the immediate 12 months. Sixteen women were not sure which method of contraception they would prefer to use after their pregnancy. Fourteen would choose LARC. Of some concern, nine would choose a combined contraception method, which is a UK Medical Eligibility Criteria Category 4 (UKMEC 4) in the first 6 weeks postpartum. ${ }^{3}$ This highlights that some women not previously using LARC would consider it, but there remained a large gap in knowledge among most of the women questioned.

Heller et al. ${ }^{1}$ found that $43 \%$ of the women they surveyed would be prepared to start LARC immediately in the postnatal period. We found that eight women were very likely, three likely and 39 not likely to accept immediate fitting of an intrauterine device/intrauterine system (IUD/IUS). In addition, nine were very likely, five likely and 36 not likely to accept immediate fitting of an implant. The reasons given for not accepting these methods included "too early", “don't feel for it", "not heard of the IUS", "not heard of either" and "no information [provided]". We therefore believe that a dedicated service in the hospital or community for postnatal women at 4 weeks would be very useful as a further opportunity to discuss contraception needs after an initial cooling-off period.

Heller et al. ${ }^{1}$ describe the importance of education in discussing LARC methods to reduce the risks of short inter-pregnancy intervals. We agree that care could be enhanced by offering antenatal and postnatal discussions about contraception, and this would improve our service. We were surprised about the general lack of knowledge about all methods of contraception, but specifically LARC. It was difficult to collect meaningful answers about the acceptability of LARC immediately postpartum.

Our survey has highlighted the need for a designated contraceptive nurse or midwife to be available to women postpartum for education and support. It has also highlighted a knowledge gap about contraception among our trainees, and we are keen to collaborate with community sexual and reproductive health teams on training opportunities.

\section{Katarina Tvarozkova}

North Middlesex University Hospital NHS Trust, London, UK, katarina.t13@gmail.com

\section{Kathryn Killicoat}

North Middlesex University Hospital NHS Trust, London, UK, kkillicoat@gmail.com

\section{Helen Munro}

St Ann's Sexual Health Centre, St Ann's Hospital, London, UK, helen.munro1@nhs.net
Abha Govind

North Middlesex University Hospital NHS Trust, London, UK, abhagovind@aol.com

Competing interests None declared.
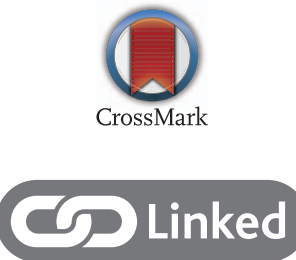

http://dx.doi.org/10.1136/jfprhc-2017-101759

J Fam Plann Reprod Health Care 2017;43:164. doi:10.1136/jprhc-2016-101562

\section{REFERENCES}

1 Heller R, Cameron S, Briggs R, et al. Postpartum contraception: a missed opportunity to prevent unintended pregnancy and short inter-pregnancy intervals. J Fam Plann Reprod Health Care 2016;42:93-98.

2 Health and Social Care Information Service. Sexual and Reproductive Health Services, England, 2014-2015. 2015. http://www.hscic.gov.uk [accessed 15 February 2017].

3 Faculty of Sexual \& Reproductive Healthcare. UK Medical Eligibility Criteria for Contraceptive Use (UKMEC 2016). Summary Table: Hormonal and Intrauterine Contraception. 2016. http://www.fsrh.org/ documents/ukmec-2016-summary-sheets/ [accessed 15 February 2017]. 